\title{
Synthesis and characterization of some novel 1,2,4-triazoles, 1,3,4-thiadiazoles and Schiff bases incorporating imidazole moiety as potential antimicrobial agents
}

\author{
MOHAMED REDA AOUAD ${ }^{1,2^{*}}$ \\ MOUSLIM MESSALI $^{1}$ \\ NADJET REZKI I,2 \\ ADEEB AL-SHEIKH ALI ${ }^{1}$ \\ ALAIN LESIMPLE 3 \\ ${ }^{1}$ Department of Chemistry \\ Faculty of Sciences, Taibah University \\ Al-Madinah Al-Munawarah \\ P.O. Box 30002, Saudi Arabia \\ ${ }^{2}$ Department of Chemistry, Faculty of \\ Sciences, University of Sciences and \\ Technology Mohamed Boudiaf \\ Laboratoire de Chimie and Electrochimie \\ des Complexes Métalliques (LCECM) \\ USTO-MB, P.O. Box 1505 Oran \\ El M'nouar, Algeria \\ ${ }^{3}$ King Abdullah University of Science \\ and Technology (KAUST) \\ Saudi Arabia
}

Accepted October 27, 2014

\begin{abstract}
(1,4,5-Triphenylimidazol-2-yl-thio)butyric acid hydrazide (3) was obtained via alkylation of 1,4,5-triphenylimidazol-2thiol (1) with ethylbromobutyrate, followed by addition of hydrazine hydrate. Treatment of acid hydrazide 3 with carbon disulfide in an ethanolic potassium hydroxide solution gave the intermediate potassium dithiocarbazinate salt, which was cyclized to 4-amino-5-[(1,4,5-triphenylimidazol-2-yl)thiopropyl]-2H-1,2,4-triazole-3-thione (4) in the presence of hydrazine hydrate. Condensation of compound 3 with alkyl/arylisothiocyanate afforded the corresponding 1-[4-(1,4,5-triphenylimidazol-2-ylthio)butanoyl]-4-alkyl/arylthiosemicarbazides (5-7), which upon refluxing with sodium hydroxide, yielded the corresponding 1,2,4-triazole-3-thiols 8-10. Under acidic conditions, compounds 4-6 were converted to aminothiadiazoles 11-13. Moreover, the series of Schiff bases 14-18 were synthesized from the condensation of compound 3 with different aromatic aldehydes. The newly synthesized compounds were characterized by IR, ${ }^{1} \mathrm{H} \mathrm{NMR},{ }^{13} \mathrm{C}$ NMR and mass spectral analyses. They were also preliminarily screened for their antimicrobial activity.
\end{abstract}

Keywords: imidazole, 1,2,4-triazole, 1,3,4-thiadiazole, Schiff bases, antimicrobial activity

Imidazole nucleus has emerged as an important class of azoles used in various applications. In addition, thioimidazole nucleus constitutes the active part of several biologically active compounds with antifungal (1) and antiasthmatic (2) activity.

\footnotetext{
*Correspondence; e-mail: aouadmohamedreda@yahoo.fr
} 
Literature survey reveals that several 1,2,4-triazole derivatives have been investigated as therapeutically interesting compounds due to the large diversity of their effects, such as anticonvulsant (3), antimicrobial (4), analgesic (5), antioxidant (6), anti-inflammatory (7), pesticidal (8), insecticidal (9), herbicidal (10) and fungicidal ones (11).

Compounds bearing 1,3,4-thiadiazole moieties have displayed a number of chemotherapeutic properties including antibacterial (12), antifungal (13), antitubercular (14) and analgesic ones (15). On the other hand, Schiff bases are considered privileged structures in drug discovery, with significant antimicrobial, antiviral, antifungal, antibacterial and anti-inflammatory (16-20) activities as well as chelating properties towards various metal ions (21).

Prompted by these observations and in continuation of our search on the synthesis of heterocyclic compounds containing nitrogen, sulfur and bicyclic systems with potential biological activity (22-24), we have designed the synthesis of a novel series of imidazole derivatives. In particular, we emphasized the strategy of combining chemically different but pharmacologically compatible molecules (the imidazole nucleus and the 1,2,4-triazole, 1,3,4-thiadiazole and/or Schiff base moiety) in one frame in order to study their antifungal and antibacterial activities.

\section{EXPERIMENTAL}

\section{General}

Melting points were determined on a variable heater (Stuart, UK) Melt-temp apparatus and are uncorrected. Percoated silica gel (Kieselgel, 0.25 mm, 60 F254, Merck, Germany) were used for thin layer chromatography. A developing solvent system of ethyl acetate/ hexane $(1 / 2)$ was used and the spots were detected by ultraviolet light. NMR spectra were measured with a Bruker $400 \mathrm{MHz}\left({ }^{13} \mathrm{C}\right.$ : $\left.100 \mathrm{MHz}\right)$ spectrometer (Bruker, Switzerland). Chemical shifts are expressed in $\delta$-values (ppm) relative to TMS as an internal standard, using DMSO- $d_{6}$ as a solvent. The IR spectra were measured in potassium bromide pellets using a Perkin-Elmer 1430 series FTIR spectrometer (Perkin-Elmer, USA). Full scan accurate mass spectra (mass range from 200 to $1500 \mathrm{Da}$ ) were obtained at high resolution [100,000, full-width half-maximum (FWHM) at $400 \mathrm{~m} / \mathrm{z}]$ on a LTQ-FT Orbitrap Velos mass spectrometer (Thermo Electron $\mathrm{GmbH}$, Germany). The hybrid mass spectrometer was equipped with a heated electrospray ionization (ESI) probe for the ion max source and operated in the positive mode. The electrospray source conditions were: capillary voltage $3.75 \mathrm{kV}$, heated capillary temperature $275{ }^{\circ} \mathrm{C}$, source temperature $450{ }^{\circ} \mathrm{C}$ and S-Lens RF level $60 \%$. Nitrogen gas flows were set at 40 for the sheath gas and at 20 for the auxiliary gas, in arbitrary units. Elemental analyses were performed using an elementar Analysensysteme GmbH-Vario EL III Element Analyzer (Germany).

\section{Syntheses}

Ethyl(1,4,5-triphenylimidazol-2-yl)thiobutyrate (2). - To a solution of compound $\mathbf{1}$ (3.281 g, $10 \mathrm{mmol})$ in DMF $(15 \mathrm{~mL}), \mathrm{NaH}(10 \mathrm{mmol})$ was added, followed by the addition of ethylbromobutyrate $(1.506 \mathrm{~g}, 10 \mathrm{mmol})$. The mixture was heated under reflux for $8 \mathrm{~h}$, then cooled and poured onto crushed ice. The product was collected by filtration, washed with water, dried and recrystallized from ethanol. 
M. R. Aouad et al.: Synthesis and characterization of some novel 1,2,4-triazoles, 1,3,4-thiadiazoles and Schiff bases incorporating imidazole moiety as potential antimicrobial agents, Acta Pharm. 65 (2015) 117-132.

(1,4,5-Triphenylimidazol-2-ylthio)butyric acid hydrazide (3). - A mixture of compound 2 $(4.425 \mathrm{~g}, 10 \mathrm{mmol})$ and hydrazine hydrate $(10 \mathrm{mmol})$ in ethanol $(25 \mathrm{~mL})$ was refluxed for $10 \mathrm{~h}$. After cooling to room temperature, ethanol was removed under reduced pressure, and the product was recrystallized from ethanol.

4-Amino-5-[(1,4,5-triphenylimidazol-2-yl)thiopropyl]-2H-1,2,4-triazole-3-thione (4). - Carbon disulfide $(1.14 \mathrm{~g}, 15 \mathrm{mmol})$ was added dropwise to a solution of compound 3 (4.281 g, $10 \mathrm{mmol})$ in absolute ethanol $(30 \mathrm{~mL})$ containing potassium hydroxide $(0.84 \mathrm{~g}, 15 \mathrm{mmol})$ at $0{ }^{\circ} \mathrm{C}$. The reaction mixture was stirred at room temperature for $16 \mathrm{~h}$, and then cooled and diluted with diethyl ether. The precipitate was filtered, washed with diethyl ether and dried. The potassium dithiocarbazinate salt was obtained in nearly quantitative yield and was used without further purification since it was moisture sensitive.

Hydrazine hydrate $(20 \mathrm{mmol})$ was added to a suspension of the potassium salt of dithiocarbazinate $(10 \mathrm{mmol})$ in water $(10 \mathrm{~mL})$ and the mixture was refluxed with stirring for $4 \mathrm{~h}$. After cooling, it was diluted with water, then acidified with aqueous hydrochloric acid. The precipitate was filtered, washed with water and recrystallized from ethanol to give yellow needles.

1-[4-(1,4,5-Triphenylimidazol-2-ylthio)butanoyl]-4-phenylthiosemicarbazides (5-7). General method. - A mixture of compound $3(4.281 \mathrm{~g}, 10 \mathrm{mmol})$ and the appropriate isothiocyanate derivative $(10 \mathrm{mmol})$ was refluxed in ethanol for $6 \mathrm{~h}$. The solution was cooled and a white solid appeared. The obtained precipitate was filtered and recrystallized from ethanol to afford the desired product.

4-Alkyl/aryl-5-[(1,4,5-triphenylimidazol-2-yl)thiopropyl]-2H-1,2,4-triazole-3-thione (8-10). General method. - A solution of the corresponding thiosemicarbazide 5-7 (10 mmol) in $100 \mathrm{~mL}$ of $4 \mathrm{~mol} \mathrm{~L}^{-1} \mathrm{NaOH}$ was refluxed for $8 \mathrm{~h}$. The resulting solution was cooled to room temperature and acidified with $37 \% \mathrm{HCl}$. The precipitate formed was filtered, washed with water and recrystallized from ethanol to afford the desired product.

5-[(1,4,5-Triphenylimidazol-2-yl)thiopropyl]-2-(N-alkyl/arylamino)-1,3,4-thiadiazoles (11-13). General method. - A mixture of the corresponding thiosemicarbazide 5-7 (10 mmol) in cold concentrated sulfuric acid $(20 \mathrm{~mL})$ was stirred for $30 \mathrm{~min}$. The mixture was then allowed to cool to room temperature. After stirring for additional $3 \mathrm{~h}$, the resulting solution was poured into ice-cold water and made alkaline to $\mathrm{pH} 8$ with ammonia. The precipitated product was filtered and recrystallized from ethanol to afford the desired product.

4-[(1,4,5-Triphenylimidazol-2-yl)thio]-N'-(arylidene)-butanehydrazides (14-18). General method. - A mixture of compound $3(0.428 \mathrm{~g}, 1 \mathrm{mmol})$ and the appropriate aromatic aldehyde $(1$ $\mathrm{mmol})$ in ethanol $(20 \mathrm{~mL})$ and $(0.5 \mathrm{~mL})$ of $\mathrm{HCl}(37 \%)$ was refluxed for $4-6 \mathrm{~h}$. After cooling, the obtained product was collected and recrystallized from ethanol.

Physical, analytical data and spectral data (MS, IR, ${ }^{1} \mathrm{H}$ - and ${ }^{13} \mathrm{C}-$ ) for the newly synthesized products $\mathbf{2 - 1 8}$ are collected in Tables I and II. Elemental analyses of all compounds were within $\pm 0.4 \%$ of the theoretical values.

\section{Antimicrobial susceptibility testing}

The clinical isolate tested in this study were obtained from the culture collection maintained at the RCMB (Regional Center for Mycology and Biotechnology/Antimicrobial unit test organisms), Al Azhar University, Cairo-Egypt. All compounds were tested in vitro 
M. R. Aouad et al.: Synthesis and characterization of some novel 1,2,4-triazoles, 1,3,4-thiadiazoles and Schiff bases incorporating imidazole moiety as potential antimicrobial agents, Acta Pharm. 65 (2015) 117-132.

Table I. Physical and analytical data for the newly synthesized compounds 2-18

\begin{tabular}{|c|c|c|c|c|c|}
\hline \multirow{2}{*}{ Compd. } & \multirow{2}{*}{$\begin{array}{l}\text { M.p. }\left({ }^{\circ} \mathrm{C}\right) / \\
\text { yield }(\%)\end{array}$} & \multirow{2}{*}{$\begin{array}{c}\text { Theor. }(\mathrm{M}+\mathrm{H}) \\
\text { Exp. MS : }[\mathrm{M}+\mathrm{H}]^{+}\end{array}$} & \multicolumn{3}{|c|}{ Calcd./found (\%) } \\
\hline & & & $\mathrm{C}$ & $\mathrm{H}$ & $\mathrm{N}$ \\
\hline 2 & $\begin{array}{c}115-116 \\
(85)\end{array}$ & $\begin{array}{l}443.18 \\
443.18\end{array}$ & $73.27 / 73.03$ & $5.92 / 5.69$ & $6.33 / 6.23$ \\
\hline 3 & $\begin{array}{c}169-170 \\
(82)\end{array}$ & $\begin{array}{l}429.18 \\
429.18\end{array}$ & $70.07 / 69.84$ & $5.64 / 5.35$ & 13.07/13.19 \\
\hline 4 & $\begin{array}{c}263-264 \\
(77)\end{array}$ & $\begin{array}{l}485.18 \\
485.18\end{array}$ & $64.43 / 64.25$ & $4.99 / 5.16$ & 17.34/17.13 \\
\hline 5 & $\begin{array}{c}238-239 \\
(90)\end{array}$ & $\begin{array}{l}564.19 \\
564.19\end{array}$ & $68.18 / 67.99$ & $5.19 / 5.01$ & $12.42 / 12.30$ \\
\hline 6 & $\begin{array}{c}190-191 \\
(83)\end{array}$ & $\begin{array}{l}516.19 \\
516.19\end{array}$ & $65.21 / 65.47$ & $5.67 / 5.39$ & $13.58 / 13.34$ \\
\hline 7 & $\begin{array}{c}222-223 \\
(80)\end{array}$ & $\begin{array}{l}502.17 \\
502.17\end{array}$ & $64.64 / 64.97$ & $5.42 / 5.14$ & 13.96/13.71 \\
\hline 8 & $\begin{array}{c}261-262 \\
(84)\end{array}$ & $\begin{array}{l}546.18 \\
546.18\end{array}$ & $70.43 / 70.31$ & $4.99 / 5.12$ & $12.83 / 12.56$ \\
\hline 9 & $\begin{array}{c}233-234 \\
(79)\end{array}$ & $\begin{array}{l}498.18 \\
498.18\end{array}$ & $67.57 / 67.42$ & $5.47 / 5.26$ & $14.07 / 13.76$ \\
\hline 10 & $\begin{array}{c}250-251 \\
(82)\end{array}$ & $\begin{array}{l}484.16 \\
484.16\end{array}$ & $67.05 / 66.79$ & $5.21 / 5.49$ & $14.48 / 14.76$ \\
\hline 11 & $\begin{array}{c}279-280 \\
(74)\end{array}$ & $\begin{array}{l}546.18 \\
546.18\end{array}$ & $70.43 / 70.17$ & $4.99 / 5.29$ & $12.83 / 12.99$ \\
\hline 12 & $\begin{array}{c}247-248 \\
(70)\end{array}$ & $\begin{array}{l}498.18 \\
498.18\end{array}$ & $67.57 / 67.91$ & $5.47 / 5.70$ & $14.07 / 14.38$ \\
\hline 13 & $\begin{array}{c}262-263 \\
(73)\end{array}$ & $\begin{array}{l}484.16 \\
484.16\end{array}$ & $67.05 / 67.29$ & $5.21 / 5.44$ & $14.48 / 14.16$ \\
\hline 14 & $\begin{array}{c}225-226 \\
(87)\end{array}$ & $\begin{array}{l}533.20 \\
533.20\end{array}$ & $72.16 / 72.45$ & $5.30 / 5.19$ & $10.52 / 10.21$ \\
\hline 15 & $\begin{array}{c}196-197 \\
(85)\end{array}$ & $\begin{array}{l}551.17 \\
551.17\end{array}$ & $69.74 / 69.39$ & $4.94 / 5.24$ & $10.17 / 10.26$ \\
\hline 16 & $\begin{array}{c}179-180 \\
(90)\end{array}$ & $\begin{array}{l}551.17 \\
551.16\end{array}$ & $69.74 / 69.95$ & $4.94 / 5.09$ & $10.17 / 10.38$ \\
\hline 17 & $\begin{array}{c}220-221 \\
(82)\end{array}$ & $\begin{array}{l}595.11 \\
595.11\end{array}$ & $64.54 / 64.87$ & $4.57 / 4.74$ & $9.41 / 9.65$ \\
\hline 18 & $\begin{array}{c}245-246 \\
(86)\end{array}$ & $\begin{array}{l}595.12 \\
595.11\end{array}$ & $64.54 / 64.78$ & $4.57 / 4.67$ & $9.41 / 9.76$ \\
\hline
\end{tabular}


Table II. IR, ${ }^{1} \mathrm{H}$-, and ${ }^{13} \mathrm{C}$ NMR spectral data for the newly synthesized compounds 2-18

\begin{tabular}{|c|c|c|c|}
\hline Compd. & $\mathrm{IR}\left(\mathrm{KBr}, v_{\max } \mathrm{cm}^{-1}\right)$ & ${ }^{1} \mathrm{H} \operatorname{NMR}(\delta, \mathrm{ppm})$ & ${ }^{13} \mathrm{C} \operatorname{NMR}(\delta, \mathrm{ppm})$ \\
\hline 2 & $\begin{array}{l}1740(\mathrm{C}=\mathrm{O}), 1637 \\
(\mathrm{C}=\mathrm{N}), 1205(\mathrm{C}-\mathrm{O})\end{array}$ & $\begin{array}{l}1.95-1.98 \text { (quin, } 2 \mathrm{H}, \\
\left.\mathrm{CH}_{2} \mathrm{CH}_{2} \mathrm{CH}_{2}\right), 2.41(\mathrm{t}, 2 \mathrm{H}, \\
\left.\mathrm{CH}_{2} \mathrm{CO}\right), 3.16\left(\mathrm{t}, 2 \mathrm{H}, \mathrm{SCH}_{2}\right), \\
4.02-4.05\left(\mathrm{q}, 2 \mathrm{H}, \mathrm{OCH} \mathrm{H}_{2}\right) \\
7.15-7.45(\mathrm{~m}, 15 \mathrm{H}, \mathrm{H}-\mathrm{Ar})\end{array}$ & $\begin{array}{l}14.09\left(\mathrm{CH}_{2} \mathrm{CH}_{3}\right), 24.61 \\
\left(\mathrm{CH}_{2} \mathrm{CH}_{2} \mathrm{CH}_{2}\right), 31.72\left(\mathrm{CH}_{2} \mathrm{CO}\right), 32.22 \\
\left(\mathrm{SCH}_{2}\right), 59.88\left(\mathrm{OCH}_{2}\right), 126.19,126.5, \\
128.17,128.28,128.35,128.92,129.13, \\
130.12,130.72,131.04,134.17,135.36, \\
137.35,142.35(\mathrm{C}-\mathrm{Ar}, \mathrm{C}=\mathrm{N})\end{array}$ \\
\hline 3 & $\begin{array}{l}3256-3380(\mathrm{NH}, \\
\left.\mathrm{NH}_{2}\right), 1692(\mathrm{C}=\mathrm{O}) \\
1630(\mathrm{C}=\mathrm{N})\end{array}$ & $\begin{array}{l}1.91-1.96 \text { (quin, } 2 \mathrm{H}, \\
\left.\mathrm{CH}_{2} \mathrm{CH}_{2} \mathrm{CH}_{2}\right), 2.14(\mathrm{t}, 2 \mathrm{H}, \\
\left.\mathrm{CH}_{2} \mathrm{CO}\right), 3.14\left(\mathrm{t}, 2 \mathrm{H}, \mathrm{SCH}_{2}\right), \\
4.16\left(\mathrm{~s}, 2 \mathrm{H}, \mathrm{NH}_{2}\right), 7.12-7.44 \\
(\mathrm{~m}, 15 \mathrm{H}, \mathrm{H}-\mathrm{Ar}), 9.00(\mathrm{~s}, 1 \mathrm{H}, \\
\mathrm{NH})\end{array}$ & $\begin{array}{l}25.33\left(\mathrm{CH}_{2} \mathrm{CH}_{2} \mathrm{CH}_{2}\right), 32.01\left(\mathrm{CH}_{2} \mathrm{CO}\right), \\
32.25\left(\mathrm{SCH}_{2}\right), 126.22,126.47,128.16, \\
128.28,128.32,128.51,128.90,129.13, \\
130.13,130.73,130.98,134.18,135.37, \\
\begin{array}{l}137.33,142.51(\mathrm{C}-\mathrm{Ar}, \mathrm{C}=\mathrm{N}), 170.89 \\
(\mathrm{C}=\mathrm{O})\end{array}\end{array}$ \\
\hline 4 & $\begin{array}{l}3240-3367(\mathrm{NH}, \\
\left.\mathrm{NH}_{2}\right), 1643(\mathrm{C}=\mathrm{N}) \\
1290(\mathrm{C}=\mathrm{S})\end{array}$ & $\begin{array}{l}\text { 2.04-2.11 (quin, } 2 \mathrm{H}, \\
\left.\mathrm{CH}_{2} \mathrm{CH}_{2} \mathrm{CH}_{2}\right), 2.82(\mathrm{t}, 2 \mathrm{H}, \\
\left.\mathrm{CH}_{2} \mathrm{C}=\mathrm{N}\right), 3.27(\mathrm{t}, 2 \mathrm{H}, \mathrm{SCH})_{2} \\
5.76\left(\mathrm{~s}, 2 \mathrm{H}, \mathrm{NH}_{2}\right), 7.22-7.46(\mathrm{~m}, \\
15 \mathrm{H}, \mathrm{H}-\mathrm{Ar}), 14.40(\mathrm{~s}, 1 \mathrm{H}, \mathrm{NH})\end{array}$ & $\begin{array}{l}23.58\left(\mathrm{CH}_{2} \mathrm{CH}_{2} \mathrm{CH}_{2}\right), 25.10 \\
\left(\mathrm{CH}_{2} \mathrm{C}=\mathrm{N}\right), 32.11\left(\mathrm{SCH}_{2}\right), 126.80, \\
126.91,127.54,128.22,128.42,128.59, \\
128.91,129.24,129.53,130.76,131.57, \\
131.57,134.57,141.90,142.08,163.38 \\
(C-\mathrm{Ar}, \mathrm{C}=\mathrm{N}), 177.64(\mathrm{C}=\mathrm{S})\end{array}$ \\
\hline 5 & $\begin{array}{l}3210-3352(\mathrm{NH}) \\
1670(\mathrm{C}=\mathrm{O}), 1642 \\
(\mathrm{C}=\mathrm{N}), 1302(\mathrm{C}=\mathrm{S})\end{array}$ & $\begin{array}{l}1.96-2.03(\text { quin, } 2 \mathrm{H}, \\
\left.\mathrm{CH}_{2} \mathrm{CH}_{2} \mathrm{CH}_{2}\right), 2.32(\mathrm{t}, 2 \mathrm{H}, \\
\left.\mathrm{CH}_{2} \mathrm{CO}\right), 3.21\left(\mathrm{t}, 2 \mathrm{H}, \mathrm{SCH}_{2}\right), \\
7.12-7.47(\mathrm{~m}, 2 \mathrm{H}, \mathrm{H}-\mathrm{Ar}), 9.57 \\
(\mathrm{~d}, 1 \mathrm{H}, \mathrm{NH}), 9.62(\mathrm{~s}, 1 \mathrm{H}, \mathrm{NH}), \\
9.90(\mathrm{~d}, 1 \mathrm{H}, \mathrm{NH})\end{array}$ & $\begin{array}{l}24.64\left(\mathrm{CH}_{2} \mathrm{CH}_{2} \mathrm{CH}_{2}\right), 31.95\left(\mathrm{CH}_{2} \mathrm{CO}\right), \\
32.12\left(\mathrm{SCH}_{2}\right), 126.20,126.45,128.13, \\
128.24,128.30,128.49,128.89,129.12, \\
\text { 130.06, 130.68, 130.95, 134.13, 135.31, } \\
\begin{array}{l}137.31,142.49(\mathrm{C}-\mathrm{Ar}, \mathrm{C}=\mathrm{N}), 173.66 \\
(\mathrm{C}=\mathrm{O})\end{array}\end{array}$ \\
\hline 6 & $\begin{array}{l}3240-3367(\mathrm{NH}) \\
1689(\mathrm{C}=\mathrm{O}), 1634 \\
(\mathrm{C}=\mathrm{N}), 1290(\mathrm{C}=\mathrm{S})\end{array}$ & $\begin{array}{l}1.03\left(\mathrm{t}, 3 \mathrm{H}, \mathrm{CH}_{2} \mathrm{CH}_{3}\right), 1.91-1.98 \\
\left.\text { (quin, } 2 \mathrm{H}, \mathrm{CH}_{2} \mathrm{CH}_{2} \mathrm{CH}_{2}\right), 2.27 \\
\left.\text { (t, } 2 \mathrm{H}, \mathrm{CH}_{2} \mathrm{CO}\right), 3.18(\mathrm{t}, 2 \mathrm{H}, \\
\left.\mathrm{SCH} \mathrm{H}_{2}\right), 3.36-3.42(\mathrm{~m}, 2 \mathrm{H}, \\
\left.\mathrm{NCH}_{2}\right), 7.16-7.45(\mathrm{~m}, 15 \mathrm{H}, \\
\mathrm{H}-\mathrm{Ar}), 7.91(\mathrm{t}, 1 \mathrm{H}, \mathrm{NH}), 9.06 \\
(\mathrm{~d}, 1 \mathrm{H}, \mathrm{NH}), 9.65(\mathrm{~s}, 1 \mathrm{H}, \mathrm{NH})\end{array}$ & $\begin{array}{l}14.45\left(\mathrm{CH}_{2} \mathrm{CH}_{3}\right), 24.60 \\
\left(\mathrm{CH}_{2} \mathrm{CH}_{2} \mathrm{CH}_{2}\right), 31.92\left(\mathrm{CH}_{2} \mathrm{CO}\right), 32.03 \\
\left(\mathrm{SCH}_{2}\right), 38.35\left(\mathrm{NCH}_{2}\right), 126.19,126.45, \\
128.13,128.23,128.31,128.49,128.90, \\
129.12,130.05,130.68,130.95,134.11, \\
135.31,137.30,142.48(\mathrm{C}-\mathrm{Ar}, \mathrm{C}=\mathrm{N}), \\
171.31(\mathrm{C}=\mathrm{O})\end{array}$ \\
\hline 7 & $\begin{array}{l}3234-3380(\mathrm{NH}) \\
1663(\mathrm{C}=\mathrm{O}), 1650 \\
(\mathrm{C}=\mathrm{N}), 1303(\mathrm{C}=\mathrm{S})\end{array}$ & $\begin{array}{l}1.92-1.99 \text { (quin, 2H, } \\
\left.\mathrm{CH}_{2} \mathrm{CH}_{2} \mathrm{CH}_{2}\right), 2.27(\mathrm{t}, 2 \mathrm{H}, \\
\left.\mathrm{CH}_{2} \mathrm{CO}\right), 2.84\left(\mathrm{~d}, 3 \mathrm{H}, \mathrm{NCH}_{3}\right), \\
3.18\left(\mathrm{t}, 2 \mathrm{H}, \mathrm{SCH} \mathrm{CH}_{2}\right), 7.14-7.45(\mathrm{~m}, \\
15 \mathrm{H}, \mathrm{H}-\mathrm{Ar}), 7.88(\mathrm{~d}, 1 \mathrm{H}, \mathrm{NH}), \\
9.12(\mathrm{~d}, 1 \mathrm{H}, \mathrm{NH}), 9.67(\mathrm{~s}, 1 \mathrm{H}, \\
\mathrm{NH})\end{array}$ & $\begin{array}{l}24.55\left(\mathrm{CH}_{2} \mathrm{CH}_{2} \mathrm{CH}_{2}\right), 30.81\left(\mathrm{NCH}_{3}\right), \\
31.91\left(\mathrm{CH}_{2} \mathrm{CO}\right), 32.01\left(\mathrm{SCH}_{2}\right), 126.20, \\
126.46,128.14,128.24,128.30,128.50, \\
\text { 128.90, 129.13, 130.04, 130.67, 130.95, } \\
\text { 134.11, 135.30, 137.30, 142.49 (C -Ar, } \\
\mathrm{C}=\mathrm{N}), 171.37(\mathrm{C}=\mathrm{O})\end{array}$ \\
\hline 8 & $\begin{array}{l}3224-3290(\mathrm{NH}), \\
1697(\mathrm{C}=\mathrm{N}), 1282 \\
(\mathrm{C}=\mathrm{S})\end{array}$ & $\begin{array}{l}1.92-1.99 \text { (quin, } 2 \mathrm{H}, \\
\left.\mathrm{CH}_{2} \mathrm{CH}_{2} \mathrm{CH}_{2}\right), 2.53(\mathrm{t}, 2 \mathrm{H}, \\
\left.\mathrm{CH}_{2} \mathrm{C}=\mathrm{N}\right), 3.12(\mathrm{t}, 2 \mathrm{H}, \mathrm{SCH})_{2} \\
7.14-7.52(\mathrm{~m}, 2 \mathrm{H}, \mathrm{H}-\mathrm{Ar}), 13.74 \\
(\mathrm{~s}, 1 \mathrm{H}, \mathrm{NH})\end{array}$ & $\begin{array}{l}24.03\left(\mathrm{CH}_{2} \mathrm{CH}_{2} \mathrm{CH}_{2}\right), 25.19 \\
\left(\mathrm{CH}_{2} \mathrm{C}=\mathrm{N}\right), 31.34\left(\mathrm{SCH}_{2}\right), 126.15, \\
126.43,128.15,128.22,128.30,128.50, \\
128.85,129.07,129.34,130.04,130.94, \\
133.64,134.07,135.25,137.29,142.13, \\
151.54,167.65(\mathrm{C}-\mathrm{Ar}, \mathrm{C}=\mathrm{N})\end{array}$ \\
\hline
\end{tabular}




\begin{tabular}{|c|c|c|c|}
\hline Compd. & $\mathrm{IR}\left(\mathrm{KBr}, v_{\max } \mathrm{cm}^{-1}\right)$ & ${ }^{1} \mathrm{H} \operatorname{NMR}(\delta, \mathrm{ppm})$ & ${ }^{13} \mathrm{C} \operatorname{NMR}(\delta, \mathrm{ppm})$ \\
\hline 9 & $\begin{array}{l}3265-3305(\mathrm{NH}), \\
1694(\mathrm{C}=\mathrm{N}), 1305 \\
(\mathrm{C}=\mathrm{S})\end{array}$ & $\begin{array}{l}1.15\left(\mathrm{t}, 3 \mathrm{H}, \mathrm{CH}_{2} \mathrm{CH}_{3}\right), 2.08-2.15 \\
\left.\text { (quin, } 2 \mathrm{H}, \mathrm{CH}_{2} \mathrm{CH}_{2} \mathrm{CH}_{2}\right), 2.80 \\
\left.\text { (t, } 2 \mathrm{H}, \mathrm{CH}_{2} \mathrm{C}=\mathrm{N}\right), 3.27(\mathrm{t}, 2 \mathrm{H}, \\
\left.\mathrm{SCH} \mathrm{H}_{2}\right), 3.89-3.94(\mathrm{q}, 2 \mathrm{H}, \mathrm{NCH}) \text { ) } \\
7.17-7.43(\mathrm{~m}, 15 \mathrm{H}, \mathrm{H}-\mathrm{Ar}), 13.56 \\
(\mathrm{~s}, 1 \mathrm{H}, \mathrm{NH})\end{array}$ & $\begin{array}{l}\text { 13.37 }\left(\mathrm{CH}_{2} \mathrm{CH}_{3}\right), 23.27 \\
\left(\mathrm{CH}_{2} \mathrm{CH}_{2} \mathrm{CH}_{2}\right), 25.56\left(\mathrm{CH}_{2} \mathrm{C}=\mathrm{N}\right), \\
31.90\left(\mathrm{SCH}_{2}\right), 48.56\left(\mathrm{NCH}_{2}\right), 126.36, \\
126.82,128.23,128.53,129.13,129.56, \\
130.60,130.71,131.23,131.76,134.08, \\
135.66,138.12,142.09,151.90,166.58 \\
(\mathrm{C}-\mathrm{Ar}, \mathrm{C}=\mathrm{N})\end{array}$ \\
\hline 10 & $\begin{array}{l}3220-3279(\mathrm{NH}) \\
1642(\mathrm{C}=\mathrm{N}), 1295 \\
(\mathrm{C}=\mathrm{S})\end{array}$ & $\begin{array}{l}\text { 2.07-2.14 (quin, } 2 \mathrm{H}, \\
\left.\mathrm{CH}_{2} \mathrm{CH}_{2} \mathrm{CH}_{2}\right), 2.50(\mathrm{~s}, 3 \mathrm{H}, \\
\left.\mathrm{NCH}_{3}\right), 2.78\left(\mathrm{t}, 2 \mathrm{H}, \mathrm{CH}_{2} \mathrm{C}=\mathrm{N}\right) \\
3.21\left(\mathrm{t}, 2 \mathrm{H}, \mathrm{SCH} \mathrm{H}_{2}\right), 7.14-7.45(\mathrm{~m}, \\
15 \mathrm{H}, \mathrm{H}-\mathrm{Ar}), 13.52(\mathrm{~s}, 1 \mathrm{H}, \mathrm{NH})\end{array}$ & $\begin{array}{l}23.60\left(\mathrm{CH}_{2} \mathrm{CH}_{2} \mathrm{CH}_{2}\right), 25.16\left(\mathrm{NCH}_{3}\right), \\
29.62\left(\mathrm{CH}_{2} \mathrm{C}=\mathrm{N}\right), 31.53\left(\mathrm{SCH}_{2}\right), \\
126.10,126.44,128.18,128.33,128.50, \\
128.67,129.09,130.04,130.69,131.03, \\
134.05,135.29,137.28,142.20,151.90, \\
166.58(\mathrm{C}-\mathrm{Ar}, \mathrm{C}=\mathrm{N})\end{array}$ \\
\hline 11 & $\begin{array}{l}3254-3298(\mathrm{NH}) \\
1634(\mathrm{C}=\mathrm{N})\end{array}$ & $\begin{array}{l}\text { 2.03-2.11 (quin, 2H, } \\
\left.\mathrm{CH}_{2} \mathrm{CH}_{2} \mathrm{CH}_{2}\right), 2.99(\mathrm{t}, 2 \mathrm{H}, \\
\left.\mathrm{CH}_{2} \mathrm{C}=\mathrm{N}\right), 3.24(\mathrm{t}, 2 \mathrm{H}, \mathrm{SCH})_{2} \\
7.21-7.59(\mathrm{~m}, 20 \mathrm{H}, \mathrm{H}-\mathrm{Ar}), 10.48 \\
(\mathrm{~s}, 1 \mathrm{H}, \mathrm{NH})\end{array}$ & $\begin{array}{l}27.81\left(\mathrm{CH}_{2} \mathrm{CH}_{2} \mathrm{CH}_{2}\right), 28.70 \\
\left(\mathrm{CH}_{2} \mathrm{C}=\mathrm{N}\right), 33.11\left(\mathrm{SCH}_{2}\right), 116.22, \\
125.86,126.56,126.85,127.23,128.23, \\
128.69,129.36,130.04,130.73,132.30, \\
134.03,140.68,141.50,141.96,147.98, \\
158.75,164.02(\mathrm{C}-\mathrm{Ar}, \mathrm{C}=\mathrm{N})\end{array}$ \\
\hline 12 & $\begin{array}{l}3213-3296(\mathrm{NH}) \\
1637(\mathrm{C}=\mathrm{N})\end{array}$ & $\begin{array}{l}1.20\left(\mathrm{t}, 3 \mathrm{H}, \mathrm{CH}_{2} \mathrm{CH}_{3}\right), 2.04-2.11 \\
\left.\text { (quin, } 2 \mathrm{H}, \mathrm{CH}_{2} \mathrm{CH}_{2} \mathrm{CH}_{2}\right), 2.97 \\
\left(\mathrm{t}, 2 \mathrm{H}, \mathrm{CH}_{2} \mathrm{C}=\mathrm{N}\right), 3.24(\mathrm{t}, 2 \mathrm{H}, \\
\left.\mathrm{SCH} \mathrm{H}_{2}\right), 3.33-3.42(\mathrm{q}, 2 \mathrm{H}, \\
\left.\mathrm{NCH}_{2}\right), 7.19-7.54(\mathrm{~m}, 15 \mathrm{H}, \\
\mathrm{H}-\mathrm{Ar}), 9.96(\mathrm{~s}, 1 \mathrm{H}, \mathrm{NH})\end{array}$ & $\begin{array}{l}\text { 13.34 }\left(\mathrm{CH}_{2} \mathrm{CH}_{3}\right), 27.78 \\
\left(\mathrm{CH}_{2} \mathrm{CH}_{2} \mathrm{CH}_{2}\right), 28.16\left(\mathrm{CH}_{2} \mathrm{C}=\mathrm{N}\right), \\
32.17\left(\mathrm{SCH}_{2}\right), 40.83\left(\mathrm{NCH}_{2}\right), 125.96, \\
\text { 126.33, 128.23, 128.64, 129.01, 129.30, } \\
\text { 129.65, 130.65, 131.03, 131.93, 134.33, } \\
\text { 142.11, 147.28, 157.74, 167.74 (C-Ar, } \\
\mathrm{C}=\mathrm{N})\end{array}$ \\
\hline 13 & $\begin{array}{l}3207-3231(\mathrm{NH}) \\
1641(\mathrm{C}=\mathrm{N})\end{array}$ & $\begin{array}{l}2.05-2.12(\text { quin, } 2 \mathrm{H}, \\
\left.\mathrm{CH}_{2} \mathrm{CH}_{2} \mathrm{CH}_{2}\right), 2.96-2.99(\mathrm{~m}, \\
\left.5 \mathrm{H}, \mathrm{CH}_{2} \mathrm{C}=\mathrm{N}, \mathrm{NCH}_{3}\right), 3.24(\mathrm{t}, \\
\left.2 \mathrm{H}, \mathrm{SCH}_{2}\right), 7.19-7.53(\mathrm{~m}, 15 \mathrm{H}, \\
H-\mathrm{Ar}), 9.90(\mathrm{~s}, 1 \mathrm{H}, \mathrm{NH})\end{array}$ & $\begin{array}{l}27.79\left(\mathrm{CH}_{2} \mathrm{CH}_{2} \mathrm{CH}_{2}\right), 28.17 \\
\left(\mathrm{CH}_{2} \mathrm{C}=\mathrm{N}\right), 32.07\left(\mathrm{SCH}_{2}\right), 38.83 \\
\left(\mathrm{NCH}_{3}\right), 125.67,126.25,128.23, \\
128.64,128.97,129.29,129.62,130.64, \\
131.22,131.88,134.52,142.23,146.77, \\
157.85,168.75(\mathrm{C}-\mathrm{Ar}, \mathrm{C}=\mathrm{N})\end{array}$ \\
\hline 14 & $\begin{array}{l}3254-3342(\mathrm{NH}), \\
1704(\mathrm{C}=\mathrm{O}), 1672 \\
(\mathrm{C}=\mathrm{N})\end{array}$ & $\begin{array}{l}1.95-2.03 \text { (quin, } 2 \mathrm{H}, \\
\left.\mathrm{CH}_{2} \mathrm{CH}_{2} \mathrm{CH}_{2}\right), 2.36(\mathrm{t}, 0.8 \mathrm{H}, \\
\left.\mathrm{CH}_{2} \mathrm{CO}\right), 2.76\left(\mathrm{t}, 1.2 \mathrm{H}, \mathrm{CH}_{2} \mathrm{CO}\right), \\
3.17-3.23(\mathrm{~m}, 2 \mathrm{H}, \mathrm{SCH})_{2} \\
7.14-8.07(\mathrm{~m}, 2 \mathrm{H}, \mathrm{H}-\mathrm{Ar}, \mathrm{OH}), \\
8.10(\mathrm{~s}, 0.25 \mathrm{H}, \mathrm{H}-\mathrm{C}=\mathrm{N}), 8.31(\mathrm{~s}, \\
0.25 \mathrm{H}, \mathrm{H}-\mathrm{C}=\mathrm{N}), 8.49(\mathrm{~s}, 0.15 \mathrm{H}, \\
\mathrm{H}-\mathrm{C}=\mathrm{N}), 8.70(\mathrm{~s}, 0.4 \mathrm{H}, \mathrm{H}-\mathrm{C}=\mathrm{N}), \\
11.37(\mathrm{~s}, 0.35 \mathrm{H}, \mathrm{CONH}), 11.49 \\
(\mathrm{~s}, 0.25 \mathrm{H}, \mathrm{CONH}), 11.54(\mathrm{~s}, \\
0.25 \mathrm{H}, \mathrm{CONH}), 11.67(\mathrm{~s}, 0.15 \mathrm{H}, \\
\mathrm{CONH})\end{array}$ & $\begin{array}{l}\text { 24.24, } 24.89\left(\mathrm{CH}_{2} \mathrm{CH}_{2} \mathrm{CH}_{2}\right), 30.56, \\
31.76\left(\mathrm{CH}_{2} \mathrm{CO}\right), 31.88,32.09\left(\mathrm{SCH}_{2}\right), \\
\text { 122.17, 123.11, 125.70, 126.13, 126.17, } \\
\text { 126.41, 127.26, 128.10, 128.46, 128.84, } \\
\text { 129.07, 130.65, 130.92, 131.13, 132.94, } \\
\text { 134.11, 135.35, 135.96, 137.29, 140.88, } \\
\text { 144.04, 160.56 (C- Ar, } \mathrm{C}=\mathrm{N}), 168.18, \\
173.82(\mathrm{C}=\mathrm{O})\end{array}$ \\
\hline
\end{tabular}




\begin{tabular}{|c|c|c|c|}
\hline Compd. & $\operatorname{IR}\left(\mathrm{KBr}, v_{\max }, \mathrm{cm}^{-1}\right)$ & ${ }^{1} \mathrm{H}$ NMR $(\delta, \mathrm{ppm})$ & ${ }^{13} \mathrm{C}$ NMR $(\delta, \mathrm{ppm})$ \\
\hline 15 & $\begin{array}{l}3260-3322(\mathrm{NH}), \\
1698(\mathrm{C}=\mathrm{O}), 1645 \\
(\mathrm{C}=\mathrm{N})\end{array}$ & $\begin{array}{l}1.99-2.07 \text { (quin, } 2 \mathrm{H}, \\
\left.\mathrm{CH}_{2} \mathrm{CH}_{2} \mathrm{CH}_{2}\right), 2.39(\mathrm{t}, 0.8 \mathrm{H}, \\
\left.\mathrm{CH}_{2} \mathrm{CO}\right), 2.72\left(\mathrm{t}, 1.2 \mathrm{H}, \mathrm{CH}_{2} \mathrm{CO}\right), \\
3.17-3.26(\mathrm{~m}, 2 \mathrm{H}, \mathrm{SCH})_{2}, \\
\text { 6.80-7.46 (m, 19H, H-Ar), } 8.26 \\
(\mathrm{~s}, 0.40 \mathrm{H}, \mathrm{H}-\mathrm{C}=\mathrm{N}), 8.34(\mathrm{~s}, \\
0.60 \mathrm{H}, \mathrm{H}-\mathrm{C}=\mathrm{N}), 11.30(\mathrm{~s}, 0.60 \mathrm{H}, \\
\mathrm{CONH}), 11.67(\mathrm{~s}, 0.40 \mathrm{H}, \\
\text { CONH) }\end{array}$ & $\begin{array}{l}\text { 24.21, 24.55 }\left(\mathrm{CH}_{2} \mathrm{CH}_{2} \mathrm{CH}_{2}\right), 30.58, \\
31.91\left(\mathrm{CH}_{2} \mathrm{CO}\right), 32.35,32.51\left(\mathrm{SCH}_{2}\right), \\
116.06,116.29,118.15,118.56,119.20, \\
119.59,126.41 .126 .58,126.88,128.50, \\
129.13,129.55,130.80,131.14,133.23, \\
135.05,136.49,140.77,142.38,156.31, \\
158.60(\mathrm{C}-\mathrm{Ar}, \mathrm{C}=\mathrm{N}), 167.74,172.75 \\
(\mathrm{C}=\mathrm{O})\end{array}$ \\
\hline 16 & $\begin{array}{l}3275-3330(\mathrm{NH}) \\
1690(\mathrm{C}=\mathrm{O}), 1640 \\
(\mathrm{C}=\mathrm{N})\end{array}$ & $\begin{array}{l}1.99-2.02(\text { quin, } 2 \mathrm{H}, \\
\left.\mathrm{CH}_{2} \mathrm{CH}_{2} \mathrm{CH}_{2}\right), 2.38(\mathrm{t}, 0.8 \mathrm{H}, \\
\left.\mathrm{CH}_{2} \mathrm{CO}\right), 2.73\left(\mathrm{t}, 1.2 \mathrm{H}, \mathrm{CH}_{2} \mathrm{CO}\right), \\
3.19,3.21(2 \mathrm{t}, 2 \mathrm{H}, \mathrm{SCH})_{2}, \\
7.13-7.93(\mathrm{~m}, 19 \mathrm{H}, \mathrm{H}-\mathrm{Ar}), 8.11 \\
(\mathrm{~s}, 0.40 \mathrm{H}, \mathrm{H}-\mathrm{C}=\mathrm{N}), 8.72(\mathrm{~s}, \\
0.60 \mathrm{H}, \mathrm{H}-\mathrm{C}=\mathrm{N}), 11.38(\mathrm{~s}, 0.60 \mathrm{H}, \\
\text { CONH), } 11.56(\mathrm{~s}, 0.40 \mathrm{H}, \mathrm{CONH})\end{array}$ & $\begin{array}{l}24.26\left(\mathrm{CH}_{2} \mathrm{CH}_{2} \mathrm{CH}_{2}\right), 30.86,31.90 \\
\left(\mathrm{CH}_{2} \mathrm{CO}\right), 31.88,32.10\left(\mathrm{SCH}_{2}\right), 126.13, \\
126.90,127.83,128.09,128.21,128.46, \\
129.05,130.65,130.89,131.16,134.10, \\
137.29,142.34,160.56(\mathrm{C}-\mathrm{Ar}, \mathrm{C}=\mathrm{N}), \\
160.61,173.84(\mathrm{C}=\mathrm{O})\end{array}$ \\
\hline 17 & $\begin{array}{l}3206-3287(\mathrm{NH}), \\
1700(\mathrm{C}=\mathrm{O}), 1692 \\
(\mathrm{C}=\mathrm{N})\end{array}$ & $\begin{array}{l}1.99-2.04 \text { (quin, } 2 \mathrm{H}, \\
\left.\mathrm{CH}_{2} \mathrm{CH}_{2} \mathrm{CH}_{2}\right), 2.37(\mathrm{t}, 0.8 \mathrm{H}, \\
\left.\mathrm{CH}_{2} \mathrm{CO}\right), 2.76\left(\mathrm{t}, 1.2 \mathrm{H}, \mathrm{CH}_{2} \mathrm{CO}\right), \\
3.18-3.25(\mathrm{~m}, 2 \mathrm{H}, \mathrm{SCH})_{2}, \\
7.16-8.50(\mathrm{~m}, 19 \mathrm{H}, \mathrm{H}-\mathrm{Ar}), 8.71 \\
(\mathrm{~s}, 0.40 \mathrm{H}, \mathrm{H}-\mathrm{C}=\mathrm{N}), 8.90(\mathrm{~s}, \\
0.60 \mathrm{H}, \mathrm{H}-\mathrm{C}=\mathrm{N}), 11.39(\mathrm{~s}, 0.60 \mathrm{H}, \\
\mathrm{CONH}), 11.77(\mathrm{~s}, 0.40 \mathrm{H}, \\
\text { CONH) }\end{array}$ & $\begin{array}{l}24.28,24.89\left(\mathrm{CH}_{2} \mathrm{CH}_{2} \mathrm{CH}_{2}\right), 30.55, \\
30.65\left(\mathrm{CH}_{2} \mathrm{CO}\right), 32.12,32.22\left(\mathrm{SCH}_{2}\right), \\
125.51,126.03,126.63,128.12,128.21, \\
128.74,130.40,130.66,131.23,132.27, \\
132.58,133.13,133.63,133.93,134.16, \\
140.71,143.89,159.68,160.53(\mathrm{C}-\mathrm{Ar}, \\
\mathrm{C}=\mathrm{N}), 161.87,167.34(\mathrm{C}=\mathrm{O})\end{array}$ \\
\hline 18 & $\begin{array}{l}3206-3287(\mathrm{NH}), \\
1700(\mathrm{C}=\mathrm{O}), 1692 \\
(\mathrm{C}=\mathrm{N})\end{array}$ & $\begin{array}{l}2.00-2.04(\text { quin, } 2 \mathrm{H}, \\
\left.\mathrm{CH}_{2} \mathrm{CH}_{2} \mathrm{CH}_{2}\right), 2.30(\mathrm{t}, 0.8 \mathrm{H}, \\
\left.\mathrm{CH}_{2} \mathrm{CO}\right), 2.78\left(\mathrm{t}, 1.2 \mathrm{H}, \mathrm{CH}_{2} \mathrm{CO}\right), \\
3.11-3.23(\mathrm{~m}, 2 \mathrm{H}, \mathrm{SCH})_{2}, \\
7.14-7.89(\mathrm{~m}, 19 \mathrm{H}, \mathrm{H}-\mathrm{Ar}), 8.31 \\
(\mathrm{~s}, 0.60 \mathrm{H}, \mathrm{H}-\mathrm{C}=\mathrm{N}), 8.49(\mathrm{~s}, \\
0.40 \mathrm{H}, \mathrm{H}-\mathrm{C}=\mathrm{N}), 11.50(\mathrm{~s}, 0.60 \mathrm{H}, \\
\mathrm{CONH}), 11.62(\mathrm{~s}, 0.40 \mathrm{H}, \\
\mathrm{CONH})\end{array}$ & $\begin{array}{l}\text { 24.29, } 24.85\left(\mathrm{CH}_{2} \mathrm{CH}_{2} \mathrm{CH}_{2}\right), 30.68, \\
31.90\left(\mathrm{CH}_{2} \mathrm{CO}\right), 32.06,32.86\left(\mathrm{SCH}_{2}\right), \\
\text { 123.12, 123.36, 126.14, 126.18, 126.90, } \\
\text { 127.96, 128.10, 128.24, 128.82, 129.05, } \\
\text { 130.08, 130.91, 131.27, 131.55, 132.96, } \\
\text { 133.08, 134.12, 135.35, 140.92, 142.41, } \\
\text { 142.46, 144.06 (C-Ar, C=N), 168.13, } \\
\text { 173.83 (C=O) }\end{array}$ \\
\hline
\end{tabular}

for their antibacterial activity against the following clinical bacterial strains: Gram negative bacteria, Escherichia coli (RCMB 0100520, Pseudomonas aeruginosa (RCMB 010043), and Gram positive bacteria, Streptococcus pneumonia (RCMB 010010), Bacillus subtilis (RCMB 010067) by the agar diffusion method (25) using Müller-Hinton agar medium for bacteria.

Antimicrobial susceptibility testing was performed by the agar disc-diffusion method. Sterile filter paper discs (8 $\mathrm{mm}$ diameter) were moistened with the compound solution in dimethylsulphoxide of specific concentration. Forty $\mu \mathrm{L}$ of the solution containing 200 $\mu \mathrm{g}$ of test compound or reference drug (gentamicin or ampicillin trihydrate) was loaded onto filter paper discs. Solvent was evaporated and discs were transferred to the inocu- 
lated plates. Loaded discs were carefully placed on Müller-Hinton for bacteria and Sabouraud's agar for fungi, previously inoculated separately with the test microorganisms. The plates were incubated at $37^{\circ} \mathrm{C}$ for bacteria and $28^{\circ} \mathrm{C}$ for fungi, diameters of the growth inhibition zones were measured after 24 hours in case of bacteria and after 48-96 hours in case of fungi. The antimicrobial activity was evaluated by measuring the zone of inhibition against the tested organisms and compared with that of the standards. Antimicrobial activities were expressed as the inhibition diameter zones in millimeters $(\mathrm{mm})$ and are presented in Tables III and IV. Each experiment was carried out in triplicate and the average zone of inhibition was calculated.

Moreover, compounds $\mathbf{2 - 1 8}$ were also subjected to in vitro testing of antifungal activity against the following four clinical fungal strains: Aspergillus fumigatus (RCMB 02568), Syncephalastrum racemosum (RCMB 05922), Geotrichum candidum (RCMB 05097), and Candida albicans (RCMB 05031) by agar diffusion method (26) using Sabouraud's agar medium for fungi.

Table III. Antibacterial activity of compounds 2-18 against Gram negative and Gram positive bacteria

\begin{tabular}{|c|c|c|c|c|}
\hline \multirow{3}{*}{ Compound } & \multicolumn{4}{|c|}{ Mean value of inhibition zone diameter (mm) } \\
\hline & \multicolumn{2}{|c|}{ Gram positive bacteria } & \multicolumn{2}{|c|}{ Gram negative bacteria } \\
\hline & S. pneumoniae & B. subtilis & P. aeruginosa & E. coli \\
\hline 2 & 13.0 & 15.3 & NA & 12.3 \\
\hline 3 & 13.9 & 16.0 & NA & 13.2 \\
\hline 4 & 19.4 & 24.6 & NA & 16.3 \\
\hline 5 & 19.8 & 22.4 & NA & 14.2 \\
\hline 6 & 17.2 & 18.3 & NA & 13.7 \\
\hline 7 & 12.3 & 14.2 & NA & NA \\
\hline 8 & 19.4 & 21.3 & NA & 12.7 \\
\hline 9 & 18.1 & 19.6 & NA & 11.9 \\
\hline 10 & 10.3 & 12.4 & NA & NA \\
\hline 11 & 19.1 & 20.8 & NA & 14.9 \\
\hline 12 & 17.3 & 18.7 & NA & 11.0 \\
\hline 13 & 17.9 & 19.0 & NA & 12.7 \\
\hline 14 & 20.6 & 25.4 & NA & 17.2 \\
\hline 15 & 14.4 & 18.2 & NA & 13.9 \\
\hline 16 & 13.8 & 16.5 & NA & 10.2 \\
\hline 17 & 18.2 & 19.4 & NA & 15.6 \\
\hline 18 & 13.7 & 15.0 & NA & 10.4 \\
\hline Ampicillin & 20.8 & 26.7 & - & - \\
\hline Gentamicin & - & - & 16.1 & 18.3 \\
\hline
\end{tabular}


M. R. Aouad et al.: Synthesis and characterization of some novel 1,2,4-triazoles, 1,3,4-thiadiazoles and Schiff bases incorporating imidazole moiety as potential antimicrobial agents, Acta Pharm. 65 (2015) 117-132.

Table IV. Antifungal activity of compounds 1-7 against some fungi

\begin{tabular}{ccccc}
\hline \multirow{2}{*}{ Compound } & \multicolumn{4}{c}{ Mean value of inhibition zone diameter (mm) } \\
\cline { 2 - 5 } & A. fumigatus & S. racemosum & G. candidum & C. albicans \\
\hline $\mathbf{2}$ & 14.3 & NA & 16.1 & 13.6 \\
$\mathbf{4}$ & 14.9 & NA & 16.8 & 14.2 \\
$\mathbf{5}$ & 19.3 & NA & 23.3 & 18.3 \\
$\mathbf{6}$ & 18.6 & NA & 20.6 & 18.7 \\
$\mathbf{7}$ & 16.7 & NA & 17.8 & 15.1 \\
$\mathbf{8}$ & NA & NA & NA & NA \\
$\mathbf{9}$ & 17.4 & NA & 18.3 & 16.4 \\
$\mathbf{1 0}$ & 16.8 & NA & 18.3 & 15.9 \\
$\mathbf{1 1}$ & NA & NA & NA & NA \\
$\mathbf{1 2}$ & 19.4 & NA & 20.6 & 18.2 \\
$\mathbf{1 3}$ & 15.2 & NA & 16.4 & 15.8 \\
$\mathbf{1 4}$ & 15.6 & NA & 17.8 & 16.3 \\
$\mathbf{1 5}$ & 20.1 & NA & 25.8 & 21.6 \\
$\mathbf{1 6}$ & 19.3 & NA & 20.3 & 18.3 \\
$\mathbf{1 7}$ & 13.2 & NA & 14.3 & 11.1 \\
$\mathbf{1 8}$ & 17.8 & NA & 15.8 & 14.7 \\
Amphotericin B & 13.9 & NA & 16.2 & 13.4 \\
\hline
\end{tabular}

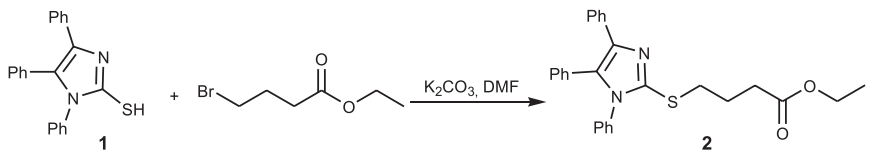

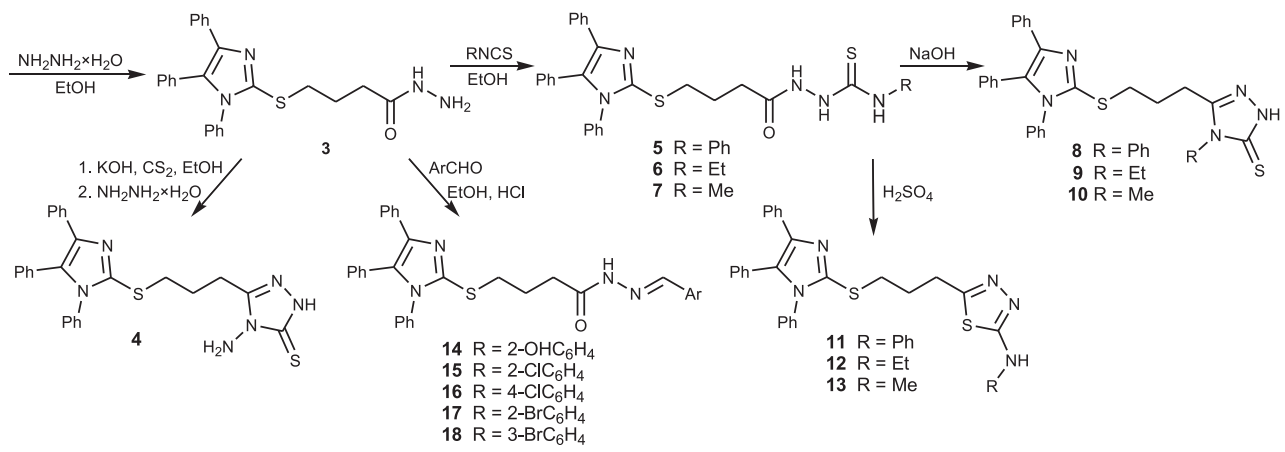

Scheme 1 
M. R. Aouad et al.: Synthesis and characterization of some novel 1,2,4-triazoles, 1,3,4-thiadiazoles and Schiff bases incorporating imidazole moiety as potential antimicrobial agents, Acta Pharm. 65 (2015) 117-132.

\section{RESULTS AND DISCUSSION}

\section{Chemistry}

Synthesis of the title azole compounds required stepwise reactions starting from 1,4,5-triphenylimidazole-2-thiol (1), as illustrated in Schemes 1-3. The starting compound 1 was prepared according to the literature (27).

Alkylation of compound 1 with 4-ethylbromobutyrate in dimethylformamide in the presence of sodium hydride as a base gave ethyl(1,4,5-triphenylimidazol-2-yl)thiobutyrate (2) in $85 \%$ yield. Hydrazinolysis of ester 2 with an equimolar amount of hydrazine hydrate furnished the corresponding acid hydrazide 3 (Scheme 1).

All the newly synthesized compounds were characterized by MS, IR, ${ }^{1} \mathrm{H}$ and ${ }^{13} \mathrm{C} \mathrm{NMR}$ spectra. The structure of compound 2 was in accord with its spectroscopic data. The IR spectrum of compound 2 showed the principal absorption band at $1740 \mathrm{~cm}^{-1}$, indicating the presence of a carbonyl group $(\mathrm{C}=\mathrm{O})$ in the molecule, which confirms the success of the alkylation reaction. In the ${ }^{1} \mathrm{H}$ NMR spectrum, the characteristic ester protons appeared as a triplet at $\delta 1.15 \mathrm{ppm}$ and a quartet at $\delta 4.02-4.05 \mathrm{ppm}$. The other methylene groups were observed as quintets ranging from $\delta 1.95-1.98 \mathrm{ppm}$, triplets at $\delta 2.41 \mathrm{ppm}$ and triplets at $\delta$ $3.16 \mathrm{ppm}$, according to $\mathrm{CH}_{2} \mathrm{CH}_{2} \mathrm{CH}_{2}, \mathrm{CH}_{2} \mathrm{CO}$ and $\mathrm{SCH}_{2}$, respectively. All aromatic protons were observed at their usual chemical shifts with $\delta 7.15-7.45 \mathrm{ppm}$. In the ${ }^{13} \mathrm{C}$ NMR spectrum, one methyl carbon, was observed at $\delta 14.09 \mathrm{ppm}$ and the carbons of the four methylene groups at $\delta 24.61,31.72,32.22$ and $59.88 \mathrm{ppm}$, respectively (see Table II). All aromatic carbons of the three phenyl groups and $\mathrm{C}=\mathrm{N}$ gave signals between $\delta 126.19-142.35 \mathrm{ppm}$. In addition, the carbon of the carbonyl group appeared at $\delta 172.38 \mathrm{ppm}$.

The IR spectrum of acid hydrazide 3 showed absorption bands at $3256-3380 \mathrm{~cm}^{-1}$ corresponding to the $\mathrm{NH}$ and $\mathrm{NH}_{2}$ groups of the acid hydrazide, which confirms the success of the hydrazinolysis reaction. The ${ }^{1} \mathrm{H}$ NMR spectrum of 3 showed a quintet around $\delta$ 1.91-1.96 ppm due to the methylene group $\mathrm{CH}_{2} \mathrm{CH}_{2} \mathrm{CH}_{2}$. The remaining methylene groups of $\mathrm{CH}_{2} \mathrm{CO}$ and $\mathrm{SCH}_{2}$ showed triplets at $\delta 2.14$ and $3.14 \mathrm{ppm}$, respectively. In addition, $\mathrm{NH}_{2}$ and $\mathrm{NH}$ groups were assigned to two singlets appearing at $\delta 4.16$ and $9.00 \mathrm{ppm}$, respectively. The structure of 3 was also illustrated from its ${ }^{13} \mathrm{C}$ NMR spectrum, which showed three carbons of the methylene groups at $\delta 25.33,32.01$ and $32.25 \mathrm{ppm}$, while the remaining aromatic carbons and $\mathrm{C}=\mathrm{N}$ appeared in the chemical shift range of $\delta 126.22-142.51 \mathrm{ppm}$. The carbon of the carbonyl group showed a signal at $\delta 170.89 \mathrm{ppm}$.

The treatment of acid hydrazide 3 with carbon disulphide in ethanolic potassium hydroxide solution led to the formation of potassium dithiocarbazinate salt. Its treatment with hydrazine hydrate under reflux furnished the desired 4-amino-5-[(1,4,5-triphenylimidazol-2-yl)thiopropyl]-2H-1,2,4-triazole-3-thione (4) in $88 \%$ yield (Scheme 1). IR spectrum of compound 4 exhibited $\mathrm{N}-\mathrm{H}$ bands in the region $3240-3367 \mathrm{~cm}^{-1}$. The absorption bands at 1643 and $1290 \mathrm{~cm}^{-1}$ are due to the presence of $\mathrm{C}=\mathrm{N}$ and $\mathrm{C}=\mathrm{S}$ stretchings of the triazole ring system, respectively. The appearance of signals approximately at $5.76 \mathrm{ppm}$ $\left(\mathrm{NH}_{2}\right)$ and $14.40 \mathrm{ppm}(\mathrm{NH})$, integrating two and one protons, respectively, in the ${ }^{1} \mathrm{H} \mathrm{NMR}$ spectra of compound 4 confirmed the conversion of 3 into the corresponding aminotriazole 4. Aromatic protons were observed at 7.22-7.46 ppm. These groups were observed at $126.80-163.38 \mathrm{ppm}$ in the ${ }^{13} \mathrm{C}$ NMR spectrum.

On the other hand, the reaction of the same hydrazide 3 with alkyl/aryl isothiocyanate furnished the corresponding aryl/alkylthiosemicarbazide derivatives 5-7 (Scheme 1). Their structures were established on the basis of their spectral data, which indicated the 
M. R. Aouad et al.: Synthesis and characterization of some novel 1,2,4-triazoles, 1,3,4-thiadiazoles and Schiff bases incorporating imidazole moiety as potential antimicrobial agents, Acta Pharm. 65 (2015) 117-132.

presence of the thiocarbonyl group $(\mathrm{C}=\mathrm{S})$ by the appearance of new absorption bands at $1290-1303 \mathrm{~cm}^{-1}$ in IR spectra. Their ${ }^{1} \mathrm{H}$ NMR spectra showed the disappearance of $\mathrm{NH}_{2}$ protons. The CONH and NHCSNH protons were observed in the range of $\delta 7.88-9.90 \mathrm{ppm}$, confirming the formation of thiosemicarbazides. Their carbon signals were observed in a chemical shift range of $\delta 171.31-173.66 \mathrm{ppm}$.

Cyclodehydration of compounds 5-7 was performed in alkaline medium to give the corresponding 4-alkyl/aryl-5-[(1,4,5-triphenylimidazol-2-yl)thiopropyl]-2H-1,2,4-triazole3-thiones (8-10) in yields ranging 79-84 \% (Scheme 1).

The structure of 1,2,4-triazoles 8-10 displayed IR, ${ }^{1} \mathrm{H}$ NMR and ${ }^{13} \mathrm{C}$ NMR spectra consistent with the assigned structures. In their IR spectra, the absorption peak corresponding to the $\mathrm{C}=\mathrm{N}$ group was observed around $1642-1697 \mathrm{~cm}^{-1}$. In their ${ }^{1} \mathrm{H}$ NMR spectra, the appearance of signals arising from the thiol group (SH) at $\delta$ 13.52-13.74 ppm provided evidence for the formation of triazole ring. Another support for the structure of these compounds was the disappearance of resonances arising from $-\mathrm{CONH}$ and $-\mathrm{NHCSNH}-$ around $\delta 7.88-9.90 \mathrm{ppm}$. The remaining protons were also observed in the expected regions (see Table II).

Ring closure of acid thiosemicarbazides 5-7 in acidic medium is a well known method for the synthesis of 1,3,4-thiadiazoles (28). Thus, 5-[(1,4,5-triphenylimidazol-2-yl) thiopropyl]-2-(N-alkyl/arylamino)-1,3,4-thiadiazoles 11-13 were obtained in 70-84 \% yield via the treatment of the above acid thiosemicarbazides 5-7 with $\mathrm{H}_{2} \mathrm{SO}_{4}$, concentration 98 $\%$, at $0{ }^{\circ} \mathrm{C}$. Their IR spectra showed the absence of $\mathrm{C}=\mathrm{O}$ and $\mathrm{C}=\mathrm{S}$ stretching bands around 1663-1670 and 1290-1302 $\mathrm{cm}^{-1}$, respectively, observed in the IR spectra of the thiosemicarbazide derivatives $5-7$. The exhibited chemical shifts obtained from their ${ }^{1} \mathrm{H}$ NMR spectra were all supported by the proposed structures of these thiadiazoles. Thus, the disappearance of CONH and NHCSNH signals and appearance of a sharp singlet at $\delta 9.90-10.48 \mathrm{ppm}$ of the NH group in the ${ }^{1} \mathrm{H}$ NMR spectra of compounds 11-13 accounted for the thiadiazole ring formation and was reconfirmed by the ${ }^{13} \mathrm{C}$ NMR peak at $\delta 164.02-168.75$ due to $\mathrm{C} 2$ of thiadiazole (see Table II).

The acid hydrazide 3 was also condensed with various aromatic aldehydes in the presence of a catalytic amount of concentrated hydrochloric acid in refluxing ethanol to afford a new series of Schiff bases 14-18 (Scheme 1).

It was reported in the literature $(29,30)$ that compounds having azomethine linkage exhibit $E / Z$ geometrical isomerism around $-\mathrm{C}=\mathrm{N}$ double bond and can exist as cis/trans amide conformers (30). Moreover, hydrazones were proven exist in higher percentage in DMSO- $d_{6}$ solution in the form of geometrical $E$ isomer.

The structures of the Schiff bases were in accord with their spectroscopic data. The IR spectra of compounds 14-17 showed the disappearance of the absorption bands of $\mathrm{NH}_{2}$ groups and the appearance of strong absorption bands at $3254-3342 \mathrm{~cm}^{-1}$ attributed to the $\mathrm{NH}$. The ${ }^{1} \mathrm{H}$ and ${ }^{13} \mathrm{C}$ NMR spectra of hydrazones 14-18 clearly showed the disappearance of the $\mathrm{NH}_{2}$ group of hydrazide structure and appearance of additional signals in the aromatic region belonging to the aldehydic protons.

The ${ }^{1} \mathrm{H}$ NMR spectra of compounds 14-18 revealed the presence of two sets of signals belonging to the $\mathrm{SCH}_{2}$ and $\mathrm{CH}_{2} \mathrm{CO}$ groups of cis and trans conformers at 2.30-2.78 and $3.17-3.26$ ppm, respectively, in a 0.4 to 0.6 ratio (Fig. 1). Moreover, the azomethine $\mathrm{CH}$ proton and the amidic NH proton for compounds 15-18 resonated as two sets of signals at 8.10-8.90 and 11.30-11.77 ppm, respectively, with the same ratio. On the other hand, the appearance of these protons in the ${ }^{1} \mathrm{H}$ NMR spectrum of compound 14 as two sets of four 


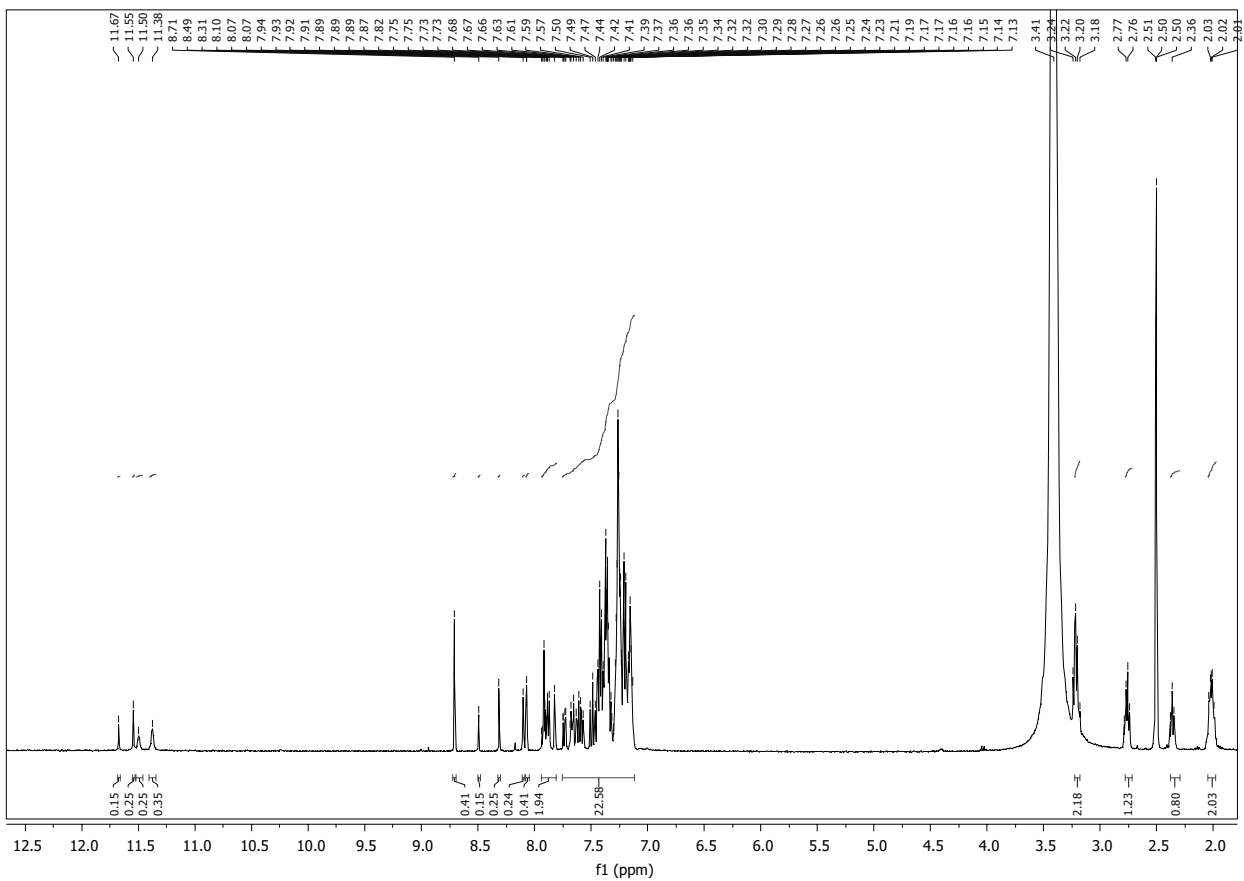

Fig. 1. ${ }^{1} \mathrm{H}$ NMR spectrum of Schiff base 14.

singlets can be considered as a proof of the formation of the geometrical $\mathrm{Z}$ form in both cis and trans conformation. This can be explained by an intramolecular hydrogen bonding connecting the azomethine nitrogen and the $\mathrm{OH}$ group of the aldehyde side chain, favoured by a stable six-member ring.

The formation of $E$ and $Z$ forms could be attributed to different steric rearrangements of hydrazone functionality in the geometric syn and anti isomers resulting from the hindered rotation of azomethine linkage.

Further evidence can be found by comparing the ${ }^{1} \mathrm{H}$ NMR spectra of hydrazide 3 with hydrazones 14-18; no conformational equilibrium, under the same experimental conditions, was observed for hydrazide 3.

Therefore, the NMR spectra of compounds $\mathbf{1 4 - 1 8}$ recorded in DMSO- $d_{6}$ solution revealed that all compounds existed as $E$ geometrical isomers, except compound $\mathbf{1 4}$ which exhibited both $E / Z$ geometrical isomerisms. Moreover, the cis/trans conformers of $E$ and $Z$ isomers were also observed.

\section{Biological activity}

Antibacterial activity. - Ethyl(1,4,5-triphenylimidazol-2-yl)thiobutyrate (2) and its hydrazide derivative 3 displayed good activity against $S$. pneumonia and B. subtilis compared 
to the ampicillin reference and comparable activity against $E$. coli compared to gentamicin reference. Triazole 4, bearing amino and thiol groups, exhibited approximately equal antimicrobial activity against all tested microorganisms except $P$. aeruginosa.

The antimicrobial activity of the series 5-7 divulged compound 5, comprising phenyl substitution at $N-4$ exhibited the highest inhibition, whereas compounds 6 and 7 with alkyl substitution showed lower activity loss of inhibition against $E$. coli for compound 7.

Evaluation of the antimicrobial activity of the synthesized 4-alkyl/aryl-5-[(1,4,5-triphenylimidazol-2-yl)thiopropyl]-1,2,4-triazole-3-thiols 8-10 revealed that compounds were more effective against Gram positive bacteria. In particular, $N-4$ phenyl substituted triazole 8 exhibited activity against tested Gram positive bacteria comparable to its ampicillin reference and lower inhibition against $E$. coli against compared to gentamicin. In contrast, compound 10, comprising methyl substitution, displayed no activity against Gram negative organisms.

The mean values of inhibition zone diameters summarized in Table III show that compounds 11-13 possess congruent antibacterial activities against the growth of S. pneumonia, B. subtilis and E. coli compared to the standards ampicillin and gentamicin. Furthermore, $N$-phenyl thiadiazole 11 exhibited the highest antibacterial activity against all tested microorganisms, which can be attributed to the presence of the thiadiazole ring.

Among the synthesized Schiff bases 14-18, compound 14, having 2-OH substitution on the phenyl ring, displayed the best antibacterial activity against S. pneumonia, B. subtilis and E. coli. However, compounds possessing unsubstituted and halosubstituted phenyl rings exhibited good to moderate activity.

\section{Antifungal activity}

In general, most of the tested compounds were found to possess significant antifungal activity against all fungal strains except $S$. racemosum.

Compounds 2 and 3 exhibited lower antifungal activity against $A$. fumigatus, G. candi$d u m$ and C. albicans compared to amphotericin B. The conversion of hydrazide structure 3 to 1,2,4-triazole functionalized with both thiol and amino groups caused congruent antifungal activity against $A$. fumigatus, G. candidum and C. albicans compared to the standard. On the other hand, 1-[4-(1,4,5-triphenylimidazol-2-ylthio)butanoyl]-4-phenyl/ethylthiosemicarbazides $(5,6)$ displayed remarkable antifungal activity, whereas their methyl analogue 7 displayed no activity against any of the fungal strains.

Contrary to what was expected, the cyclization of compounds 5-7 to their corresponding 1,2,4-triazoles (8-10) caused no enhancement of the antifungal activity. On the other hand, the antifungal activity of the 1,3,4-thiadiazoles 11-13 revealed that all the tested compounds showed significant inhibition; compound $\mathbf{1 1}$ having phenyl substitution exhibited the highest activity. Thiadiazoles 12 and $\mathbf{1 3}$ showed comparatively lower activity against $A$. fumigatus, G. candidum and C. albicans compared to the standard.

In the studied Schiff bases series, 4-[(1,4,5-triphenylimidazol-2-yl)thio]- $N^{\prime}$-(2-hydroxybenzylidene)-butanehydrazide (14) exhibited the highest antifungal activity against all fungal strains compared to amphotericin $B$. 
M. R. Aouad et al.: Synthesis and characterization of some novel 1,2,4-triazoles, 1,3,4-thiadiazoles and Schiff bases incorporating imidazole moiety as potential antimicrobial agents, Acta Pharm. 65 (2015) 117-132.

\section{CONCLUSIONS}

In conclusion, a series of novel imidazole compounds bearing 1,2,4-triazole, 1,3,4-thiadiazole or Schiff base moieties were synthesized successfully via multistep synthesis. All the new compounds were fully spectroscopically characterized. The title compounds were synthesized as new compounds with antimicrobial activity in vitro.

Acknowledgements. - We are grateful to Dr. M. M. Elaasser, Regional Center for Mycology and Biotechnology/Antimicrobial unit test organisms, Alazhar University, Cairo, Egypt, for providing the facilities to determine the antibacterial and antifungal activities.

\section{REFERENCES}

1. E. D. Weinber, Antifungal Agents. Burger's Medicinal Chemistry and Drug Discovery, $5^{\text {th }}$ ed., Wiley Interscience, New York 1996, Vol. 2, pp. 637-652.

2. U. Sahoo, S. Biswal, S. Sethy, H. K. S. Kumar and M. Banerjee, Imidazole and its biological activities: A review, Asian J. Res. Chem. 5 (2012) 171-182.

3. M. Mahdavi, T. Akbarzadeh, V. Sheibani, M. Abbasi, L. Firoozpour, S. A. Tabatabaic, A. Shafiee and A. Foroumadi, Synthesis of two novel 3-amino-5-[4-chloro-2-phenoxyphenyl]-4H-1,2,4-triazoles with anticonvulsant activity, Iranian J. Pharm. Res. 9 (2010) 265-269.

4. O. Bekircan, B. Kahveci and O. B. Ozgumus, Synthesis of some $N$-alkoxycarbonyl- $N$ "'-benzoylbenzamidrazones ( $p$-toluamidrazones) and 1,3,5-trisubstituted 1,2,4-triazole derivatives from $\mathrm{N}$ benzoylimidates and their antimicrobial and anticancer screening studies, Chin. J. Chem. 25 (2007) 1871-1877; DOI: 10.1002/cjoc.200790345.

5. M. Hamdy, A. Rahman and M. Hussein, Synthesis of $\beta$-hydroxypropanoic acid derivatives as anti-inflammatory, analgesic and antimicrobial agents, Arch. Pharm. Chem. Life Sci. 339 (2006) 378-387; DOI: 10.1002/ardp.200600016.

6. O. Bekircan, M. Kucuk, B. Kahveci and S. Kolayl1, Convenient synthesis of fused heterocyclic 1,3, 5 -triazines from some $\mathrm{N}$-acyl imidates and heterocyclic amines as anticancer and antioxidant agents, Arch. Pharm. 338 (2005) 365-372; DOI: 10.1002/ardp.200400964.

7. G. T. Zitouni, Z. A. Kaplancıklı, A. Ozdemir, P. Chevallet, H. B. Kandilci and B. Gumus, Studies on 1,2,4-triazole derivatives as potential anti-inflammatory agents, Arch. Pharm. Chem. Life Sci. 340 (2007) 586-590; DOI: 10.1002/ardp.200700134.

8. B. Chai, X. Qian, S. Cao, H. Liu and G. Song, Synthesis and insecticidal activity of 1,2,4-triazole derivatives, ARKIVOC ii (2003) 141-145.

9. M. M. Ghorab, S. G. Abdel-Hamide, G. M. Ali and E. H. Shaurub, Synthesis and insecticidal activity of some new 3-[4(3H)-quinazolinone-2-yl)thiomethyl]-1,2,4-triazole-5-thiols, Pestic. Sci. 48 (1996) 31-35; DOI: 10.1002/(SICI)1096-9063(199609)48.

10. G. Turan-Zitouni, Z. A. Kaplancıklı and A. Ozdemir, Synthesis of some triazolylthioacetamidobenzofuran derivatives, Farmaco 57 (2002) 573-578; DOI: 10.1016/S0014-827X(02)01250-8.

11. M. Chen, X.-F. Wang, S.-S. Wang, Y.-X. Feng, F. Chen and C.-L. Yang, Synthesis, characterization and fungicidal activities of novel fluorinated 3,5-disubstituted-4H-1,2,4-triazol-4-amines, J. Fluorine Chem. 135 (2012) 324 323-329; DOI: 10.1016/j.jfluchem.2011.12.015.

12. A. Foroumadi, S. Emami, A. Hassanzadeh, M. Rajaee, K. Sokhanvar, M. H. Moshafi and A. Shafiee, Synthesis and antibacterial activity of $N$-(5-benzylthio-1,3,4-thiadiazol-2-yl) and N-(5-benzylsulfonyl-1,3,4-thiadiazol-2-yl)piperazinyl quinolone derivatives, Bioorg. Med. Chem. Lett. 15 (2005) 4488-4492; DOI: 10.1016/j.bmcl.2005.07.016. 
M. R. Aouad et al.: Synthesis and characterization of some novel 1,2,4-triazoles, 1,3,4-thiadiazoles and Schiff bases incorporating imidazole moiety as potential antimicrobial agents, Acta Pharm. 65 (2015) 117-132.

13. B. Modzelewska-Banachiewicz, J. Banachiewicz, A. Chodkowska, E. Jagiello-Wojtowicz and L. Mazur, Synthesis and biological activity of new derivatives of 3-(3,4-diaryl-1,2,4-triazole-5-yl)propenoic acid, Eur. J. Med. Chem. 39 (2004) 873-877; DOI: 10.1016/j.ejmech.2004.07.002.

14. N. Solak and S. Rollas, Synthesis and antituberculosis activity of 2-(aryl/alkylamino)-5-(4-aminophenyl)-1,3,4-thiadiazoles and their Schiff bases, ARKIVOC xii (2006) 173-181; DOI: 10.3998/ ark.5550190.0007.c20.

15. S. Schenone, C. Brullo, O. Bruno, F. Bondavalli, A. Ranise, W. Filippelli, B. Rinaldi, A. Capuano and G. Falcone, New 1,3,4-thiadiazole derivatives endowed with analgesic and anti-inflammatory activities, Bioorg. Med. Chem. 14 (2006) 1698-1705; DOI: 10.1016/j.bmc.2005.10.064.

16. S. Rollas, N. Gulerman and H. Erdeniz, Synthesis and antimicrobial activity of some new hydrazones of 4-fluorobenzoic acid hydrazide and 3-acetyl-2,5-disubstituted-1,3,4-oxadiazolines, Farmaco 57 (2002) 171-174; DOI: 10.1016/S0014-827X(01)01192-2.

17. T. R. Bal, B. Anand, P. Yogeeswari and D. Sriram, Synthesis and evaluation of anti-HIV activity of isatin $\beta$-thiosemicarbazone derivatives, Bioorg. Med. Chem. Lett. 15 (2005) 4451-4455; DOI: 10.1016/j. bmcl.2005.07.046.

18. S. N. Pandeya, D. Sriram, G. Nath and E. De Clercq, Synthesis, antibacterial, antifungal and antiHIV activities of Schiff and Mannich bases derived from isatin derivatives and N-[4-(4'-chlorophenyl)thiazol-2-yl] thiosemicarbazide, Eur. J. Pharm. Sci. 9 (1999) 25-31; DOI: 10.1016/S09280987(99)00038-X.

19. S. N. Pandeya, D. Sriram, G. Nath and E. De Clercq, Synthesis, antibacterial, antifungal and antiHIV evaluation of Schiff and Mannich bases of isatin and its derivatives with triazole, Arzneim. Forsch. 50 (2000) 55-59.

20. S. M. Sondhi, M. Dinodia and A. Kumar, Synthesis, anti-inflammatory and analgesic activity evaluation of some amidine and hydrazone derivatives, Bioorg. Med. Chem. 14 (2006) 4657-4663; DOI: 10.1016/j.bmc.2006.02.014.

21. M. R. Maurya, S. Agarwal, M. Abid, A. Azam, C. Bader, M. Ebel and D. Rehder, Synthesis, characterization, reactivity and in vitro antiamoebic activity of hydrazone based oxovanadium(IV), oxovanadium(V) and $\mu$-bis(oxo)bis\{oxovanadium(V)\} complexes, Dalton Trans. (2006) 937-947; DOI: $10.1039 /$ B512326G.

22. E. S. H. El Ashry, A. A. Kassem, H. Abdel-Hamid, F. F. Louis, Sh. A. N. Khattab and M. R. Aouad, Synthesis of 4-amino-5-(3-chloro-2-benzothienyl)-3-mercapto-1,2,4-triazolo[3,4-b][1,3,4]thiadiazoles and triazolo[3,4,b][1,3,4]thiadiazines under classical and microwave conditions, ARKIVOC xiv (2006) 119-132.

23. A. H. K. Sharba, R. H. Al-Bayati, N. Rezki and M. R. Aouad, Synthesis of thiadiazoles and 1,2,4triazoles derived from cyclopropane dicarboxylic acid, Molecules 10 (2005) 1153-1160; DOI: $10.3390 / 10091153$.

24. M. R. Aouad, N. Rezki, M. Kasmi, L. Aouad and M. A. Rezki, Synthesis, characterization and evaluation of antimicrobial activity of some novel 1,2,4-triazoles and 1,3,4-thiadiazoles bearing imidazole nucleus, Heterocycles 85 (2012) 1141-1154; DOI: 10.3987/COM-12-12456.

25. European Committee for Antimicrobial Susceptibility Testing (EUCAST) of the European Society of Clinical Microbiology and Infectious Diseases (ESCMID), Determination of Minimum Inhibitory Concentrations (MICs) of Antibacterial Agents by Agar Dilution, Clin. Microbiol. Infect. (Suppl. 6) (2000) 509-515; DOI: 10.1046/j.1469-0692.2000.00142.x.

26. National Committee for Clinical Laboratory Standards, Methods for Dilution Antimicrobial Susceptibility Tests for Bacteria that Grow Aerobically. Approved Standard M7-A5, $5^{\text {th }}$ ed., NCCLS, Wayne 2000.

27. E. S. H. El Ashry, N. Rashed, L. F. Awad, E. Ramadan, S. M. Abdel-Maggeed and N. Rezki, MAOS versus conventional synthesis of 4,5-di- and 3,4,5-triphenylimidazole-2-thione and their derivatives, ARKIVOC vii (2007) 30-40. 
28. H. Kumar, S. A. Javed, S. A. Khan and M. Amir, 1,3,4-Oxadiazole/thiadiazole and 1,2,4-triazole derivatives of biphenyl-4-yloxy acetic acid: synthesis and preliminary evaluation of biological properties, Eur. J. Med. Chem. 43 (2008) 2688-2698; DOI: 10.1016/j.ejmech.2008.01.039.

29. N. Galic, B. Peric, B. Kojic-Prodic and Z. Cimerman, Structural and spectroscopic characteristics of aroylhydrazones derived from nicotinic acid hydrazide, J. Mol. Struct. 559 (2001) 187-194; DOI: 10.10.1016/S0022-2860(00)00703-1.

30. V. Klimesova, L. Zahajska, K. Waisser, J. Kaustova and U. Möllmann, Synthesis and antimycobacterial activity of 1,2,4-triazole 3-benzylsulfanyl derivatives, Farmaco 59 (2004) 279-288; DOI: 10.1016/j.farmac.2004.01.006. 